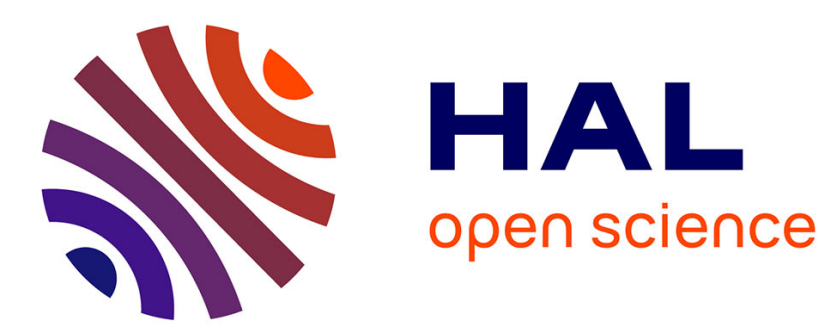

\title{
Variations dimensionnelles des propriétés électroniques de couches minces pulvérisées en champ électrique haute-fréquence \\ H. Murray
}

\section{- To cite this version:}

H. Murray. Variations dimensionnelles des propriétés électroniques de couches minces pulvérisées en champ électrique haute-fréquence. Revue de Physique Appliquée, 1975, 10 (6), pp.427-432. 10.1051/rphysap:01975001006042700 . jpa-00243941

\section{HAL Id: jpa-00243941 https://hal.science/jpa-00243941}

Submitted on 1 Jan 1975

HAL is a multi-disciplinary open access archive for the deposit and dissemination of scientific research documents, whether they are published or not. The documents may come from teaching and research institutions in France or abroad, or from public or private research centers.
L'archive ouverte pluridisciplinaire HAL, est destinée au dépôt et à la diffusion de documents scientifiques de niveau recherche, publiés ou non, émanant des établissements d'enseignement et de recherche français ou étrangers, des laboratoires publics ou privés. 


\title{
VARIATIONS DIMENSIONNELLES DES PROPRIÉTÉS ÉLECTRONIQUES DE COUCHES MINCES PULVÉRISÉES EN CHAMP ÉLECTRIQUE HAUTE-FRÉQUENCE
}

\author{
H. MURRAY \\ Laboratoire d'Electronique et d'Automatique, U. E. R. des Sciences et Techniques \\ BP 4006, 76077 Le Havre Cedex, France
}

(Reçu le 24 avril 1975, accepté le 9 juin 1975)

\begin{abstract}
Résumé. - Si les propriétés diélectriques des couches très minces de sulfure de zinc d'épaisseur comprise entre 100 et $600 \AA$ ont montré leur caractère particulièrement homogène [1] ; l'étude ultérieure de l'ensemble des propriétés électroniques (conduction et photoconduction) liées à la présence de centres coulombiens distribués dans le diélectrique met en évidence l'existence d'une zone de transition d'épaisseur voisine de $150 \AA$. Cette zone de transition présente l'intérêt particulier de provoquer un renforcement de l'effet photovoltaïque Poole-Frenkel que nous avons récemment mis en évidence [2].
\end{abstract}

\begin{abstract}
Dielectric properties of very thin zinc sulphide films between 100 and $600 \AA$ are characterized by a homogeneous dielectric growth [1]. Further data, concerning conduction and photoconduction, determined by coulombic centres distributed within the dielectric provide evidence of the existence of a transition zone $150 \AA$ in thickness in which the Poole Frenkel photovoltaic effect is amplified [2].
\end{abstract}

1. Introduction. - Les caractéristiques diélectriques des structures $\mathrm{Al}-\mathrm{ZnS}-\mathrm{Au}$, décrites dans une étude précédente [1] ont montré, à partir de la loi de corrélation entre l'épaisseur géométrique et l'épaisseur diélectrique des processus de croissance homogènes lorsque l'épaisseur est supérieure à $200 \AA \AA$. Dans cette étude, nous n'avons pu mettre en évidence des phénomènes dimensionnels dans le volume du diélectrique affectant la valeur réelle de la permittivité relative. Ce n'est qu'à partir des phénomènes de conduction et de photoconduction que nous pouvons postuler l'existence d'une épaisseur critique voisine de $150 \AA$; valeur qu'on peut rapprocher des résultats obtenus en pulvérisation continue de métaux ou d'isolants [3].

Nous en concluons, que si la pulvérisation cathodique réactive haute-fréquence permet le dépôt de couches particulièrement stables et homogènes, il s'établit néanmoins une zone de transition entre le métal de base et cette structure homogène. Le caractère partiellement lacunaire de cette zone de transition entraîne une variation qualitative des propriétés électroniques lorsque celles-ci sont essentiellement liées à des effets de volume comme nous l'avons montré lors des études de conduction [4].
Les procédés expérimentaux, précédemment décrits [1] consistent à déposer successivement les trois couches Métal $_{1}$-diélectrique-Métal ${ }_{2}$ en procédant ensuite à une stabilisation associant des cycles de variations thermiques et l'application d'un champ électrique. Le diélectrique pulvérisé à partir d'une cible de zinc dans un milieu fortement réactif (Argon et hydrogène sulfuré) présente une structure microcristalline cubique [2].

2. Identification des processus de croissance. - Le modèle macroscopique de croissance a été étudié à partir de trois critères expérimentaux [1] :

- variation de la masse déposée en fonction du temps de pulvérisation;

- variation de l'épaisseur diélectrique déduite de $1 / C$ en fonction du temps de pulvérisation ;

- variation de l'épaisseur géométrique, mesurée par interférométrie en fonction de $1 / c$.

C'est la cohérence entre ces trois lois expérimentales qui permet de conclure à une croissance homogène du diélectrique en volume, avec une valeur de la permittivité correspondant au sulfure de zinc massif [1]. 
Les capacités obtenues, après stabilisation se révèlent être particulièrement stables vis-à-vis des paramètres extérieures (tension continue appliquée, température, fréquence) et conservent cette stabilité dans le temps qu'elles aient été conservées sous vide ou séjournées à l'air ambiant.

Ce n'est qu'en étudiant la partie imaginaire de la permittivité relative où apparaît un pic de relaxation pour les faibles épaisseurs (Fig. 1) qu'il est possible de postuler l'existence d'une zone de transition, partiellement lacunaire. Néanmoins, ces résultats n'ont qu'un caractère qualitatif, et c'est en poursuivant l'étude des processus de conduction et de photoconduction, directement liés aux défauts ponctuels du modèle cristallin, que la zone de transition apparaît par renforcement de la conduction ou de l'effet photovoltaique Poole Frenkel [2].

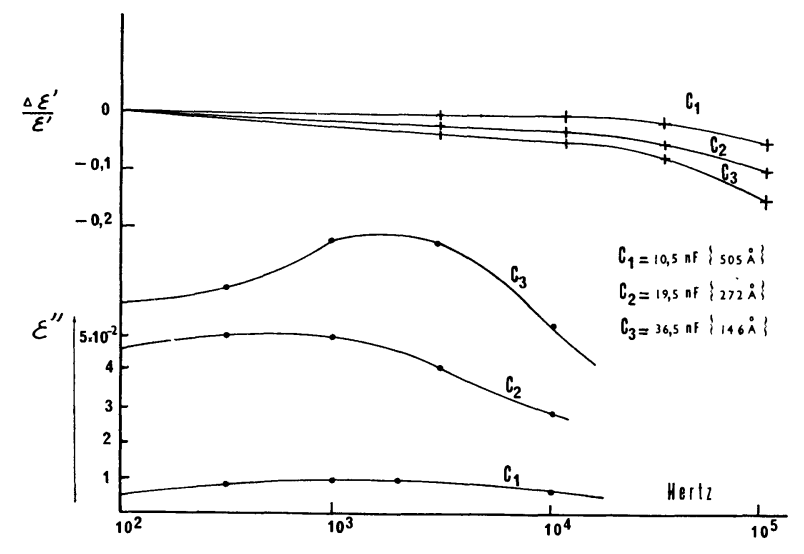

FIG. 1. - Variations de la permittivité en fonction de la fréquence.

3. Etude de la permittivité relative. - Les paramètres diélectriques des structures dont l'épaisseur est supérieur à $500 \AA$ sont comparables aux valeurs $\mathrm{du}$ sulfure de zinc massif; par contre, l'apparition d'un maximum de la permittivité imaginaire $\varepsilon^{\prime \prime}$ en fonction de la fréquence, pour les faibles épaisseurs suggère l'existence de deux couches de diélectrique.

Si on appelle $d_{1}$ l'épaisseur de la première couche et $d_{2}$ l'épaisseur de la seconde, la permittivité d'une couche d'épaisseur $d$ supérieure à $d_{1}$ est donnée par

$$
\frac{1}{\varepsilon}=\frac{d_{1}}{d_{1}+d_{2}} \cdot \frac{1}{\varepsilon_{1}}+\frac{d_{2}}{d_{1}+d_{2}} \cdot \frac{1}{\varepsilon_{2}}
$$

Si $d_{1} \gg d_{2}$

$$
\frac{1}{\varepsilon} \simeq \frac{1}{\varepsilon_{1}},
$$

les paramètres diélectriques sont pratiquement ceux de la $1^{\text {re }}$ zone. Si $d_{1} \ll d_{2}$

$$
\frac{1}{\varepsilon} \simeq \frac{1}{\varepsilon_{2}}
$$

les paramètres diélectriques, dont les valeurs expérimentales sont proches du $\mathrm{ZnS}$ massif, sont ceux de la seconde zone.

3.1 ReLAXATION Dipolaire. - L'expression générale de la permittivité diélectrique d'un milieu présentant une relaxation dipolaire est donnée par l'équation de Debye [5].

$$
\varepsilon=\varepsilon_{\infty}+\frac{\varepsilon_{\mathrm{s}}-\varepsilon_{\infty}}{1-j \omega \tau}=\varepsilon^{\prime}-j \varepsilon^{\prime \prime}
$$

où $\varepsilon_{\mathrm{s}}$ est la constante diélectrique à fréquence nulle, $\tau$ le temps de relaxation dipolaire, $\omega$ la fréquence et $\varepsilon_{\infty}$ la constante diélectrique pour $\omega \rightarrow \infty$.

Si le diélectrique ne présente qu'un seul temps de relaxation, le diagramme $\left(\varepsilon^{\prime}, \varepsilon^{\prime \prime}\right)=f(\omega)$ dans le plan complexe [6] est un demi-cercle centré sur l'axe des abcisses. En cas de distribution des temps de relaxation [7] la permittivité s'écrit :

$$
\varepsilon=\varepsilon_{\infty}+\frac{\varepsilon_{\mathrm{s}}-\varepsilon_{\infty}}{1-(j \omega \tau) 1-\alpha} \quad \text { avec } \quad 0<\alpha<1
$$

Le diagramme correspondant est seulement un arc de cercle ; il est alors difficile de l'identifier, surtout si les variations relatives sont faibles. Mais, dans tous les cas où les pertes diélectriques résultent d'un phénomène de relaxation, les mêmes variations quantitatives spécifiques sont observées :

- une décroissance de la permittivité $\varepsilon^{\prime}$ avec la pulsation,

- et un maximum de la permittivité $\varepsilon^{\prime \prime}$ lorsque la pulsation vaut $\omega=1 / \tau$.

En ce qui concerne les couches de forte épaisseur diélectrique (supérieure à $200 \AA$ ) les faibles pertes diélectriques et la lente décroissance de $\varepsilon^{\prime}$ avec $\omega$ sont compatibles avec une relaxation ionique ou électronique, dont les fréquences de relaxation sont supérieures à $1 \mathrm{MHz}$ [5].

3.2 Conclusion. - L'existence d'un maximum de $\varepsilon^{\prime \prime}$ et la décroissance plus rapide de $\varepsilon^{\prime}$ en fonction de la fréquence caractérise les épaisseurs de diélectrique proches d'une centaine d'Angströms, dimension habituelle de la zone critique de $1^{\text {re }}$ espèce ; la fréquence de relaxation comprise entre 1 et $10 \mathrm{kHz}$ se situe maintenant dans la gamme de fréquence de relaxation dipôlaire. Ce processus de dépôt des couches diélectriques permet de saisir la cohérence de ces observations. Le mode de croissance de la couche déposée pendant la première phase de condensation s'effectue toujours à partir d'un plasma contenant des molécules résiduelles, libérées par la décharge lumineuse, qui dans certaines proportions se retrouvent dans la couche mince et induisent une relaxation dipôlaire [5]. L'entraînement de toutes ces molécules par le flux gazeux crée un régime stable de condensation après quelques minutes; la deuxième phase de croissance s'effectue alors sans variations dimen- 
sionnelles, et conduit à un diélectrique homogène aux propriétés bien définies ne laissant place à aucune relaxation extrinsèque dans le domaine de fréquence étudié. Les phénomènes observés décrivent donc bien un certain aspect structurel des couches, spécifiquement lié au mode de pulvérisation. Notons que si les couches pulvérisées en haute fréquence présentent qualitativement un mode de croissance identique aux couches obtenues en champ continu [3] tout au moins dans la phase initiale de condensation, il apparaît des différences quantitatives qui sont liées aux conditions expérimentales telle que la faible pression de travail (environ $5 \times 10^{-3}$ torr) qui augmente le libre parcours moyen des atomes issus de la cible et diminue la pollution du dépôt, ce qui limite les pertes diélectriques à un niveau faible $(\operatorname{tg} \delta<0,01)$.

4. Phénomènes de conduction. - 4.1 Conduction A CHAMP ÉLEVÉ. - Les caractéristiques couranttension, linéaires dans la représentation $\log I,(V)^{1 / 2}$ correspondent à une émission d'électrons dans une bande de conduction présentant des perturbations locales liées à la présence de centres coulombiens [8].

L'expression mathématique de cette émission formulée par R. M. Hill [9] donne les variations de la densité de courant en fonction du champ électrique.

$$
J=2 e N_{i}(k T)^{4} \beta^{-2} \mu \exp -\frac{E_{\mathrm{c}}}{k T} \alpha^{2} \operatorname{sh} \alpha
$$

avec

$E_{\mathrm{c}}$ : Energie d'ionisation des centres coulombiens

$$
\beta=2\left(\frac{e^{3}}{4 \pi \varepsilon_{\mathrm{r}} \varepsilon_{0}}\right)^{1 / 2}
$$

et

$$
\alpha=\beta \frac{(E)^{1 / 2}}{k T}=1,05 \times 10^{-3}(E)^{1 / 2}
$$

$E$ : Champ électrique exprimé en volts par mètres.

Si on tient compte des valeurs élevées du champ électrique appliqué, le paramètre $\alpha$ est compris entre 2 et 10 ce qui permet d'effectuer l'approximation $\operatorname{sh} \alpha \pm \exp \alpha$, et entraîne des relations expérimentales linéaires $\log J, E^{1 / 2}$, observées par de nombreux auteurs [8].

Nous avons alors présenté le logarithme du courant $I$ en fonction de la racine carrée du champ appliqué $E$ (Fig. 2), en remarquant que le courant $I$ est proportionnel à la densité d'impuretés $N_{\mathrm{i}}$.

Cette étude appelle les remarques suivantes :

- les courbes correspondant à différentes épaisseurs de diélectrique ne sont pas confondues, mais leurs pentes ne présentent pas une dispersion supérieure à $10 \%$ autour d'une valeur cohérente avec la valeur théorique

$$
\frac{\alpha}{(E)^{1 / 2}}=\frac{2}{k T}\left(\frac{e^{3}}{4 \pi \varepsilon_{\mathrm{r}} \varepsilon_{0}}\right)^{1 / 2} \simeq 10^{-3}\left[V^{-1} \mathrm{~m}\right]^{1 / 2}
$$

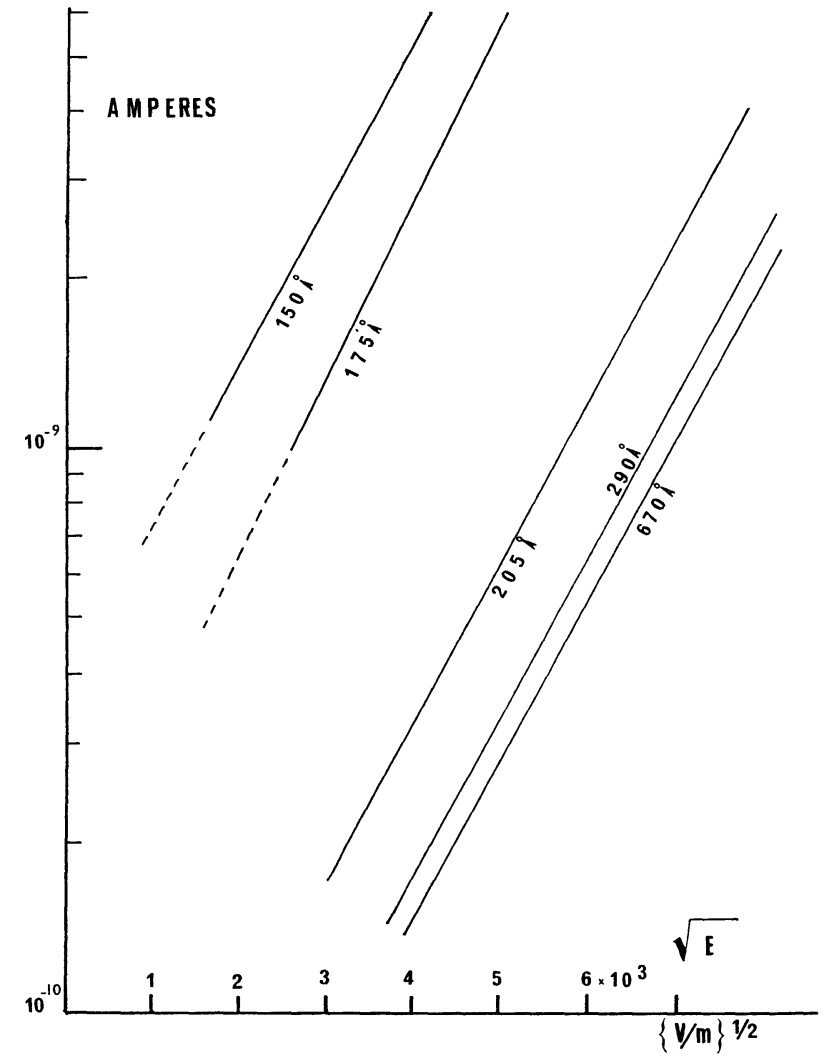

Fig. 2. - Caractéristiques $\log I, E^{1 / 2}$.

- la translation verticale des courbes $\log I$, $(E)^{1 / 2}$ en fonction de $d$ montre que la densité d'impuretés augmente lorsque l'épaisseur diminue.

Nous avons représenté (Fig. 3) le paramètre de translation des courbes $\log I, E^{1 / 2}$ en fonction de l'épaisseur ; celui-ci est proportionnel au logarithme de la densité de centres $N_{\mathrm{i}}$.

Nous distinguons sur cette courbe 3 régions :

La région $\mathrm{I}$, au-dessus de $300 \AA$ correspond à une valeur pratiquement constante de la densité d'impuretés.

La région II, entre 200 et $300 \AA$ montre une variation brusque de la densité $N_{\mathrm{i}}$.

Enfin dans la région III, au-dessous de $200 \AA$ la densité d'impuretés continue à croître avec une pente plus faible que dans la région II et caractérise la zone lacunaire.

Les hypothèses de Laville Saint-Martin [3] relatives aux processus de croissance des couches pulvérisées, qui nous ont permis de justifier l'apparition de pertes diélectriques plus importantes en dessous de $200 \AA$ prévoient l'existence d'une zone d'épaisseur critique de première espèce dont le caractère lacunaire permet l'insertion de particules étrangères. Cette zone de première espèce a une épaisseur d'environ $150 \AA$ et s'arrête lorsque le relief cesse d'être accidenté pour devenir uniforme, ce qui explique la brusque variation de $N_{\mathrm{i}}$ au voisinage de cette valeur. 


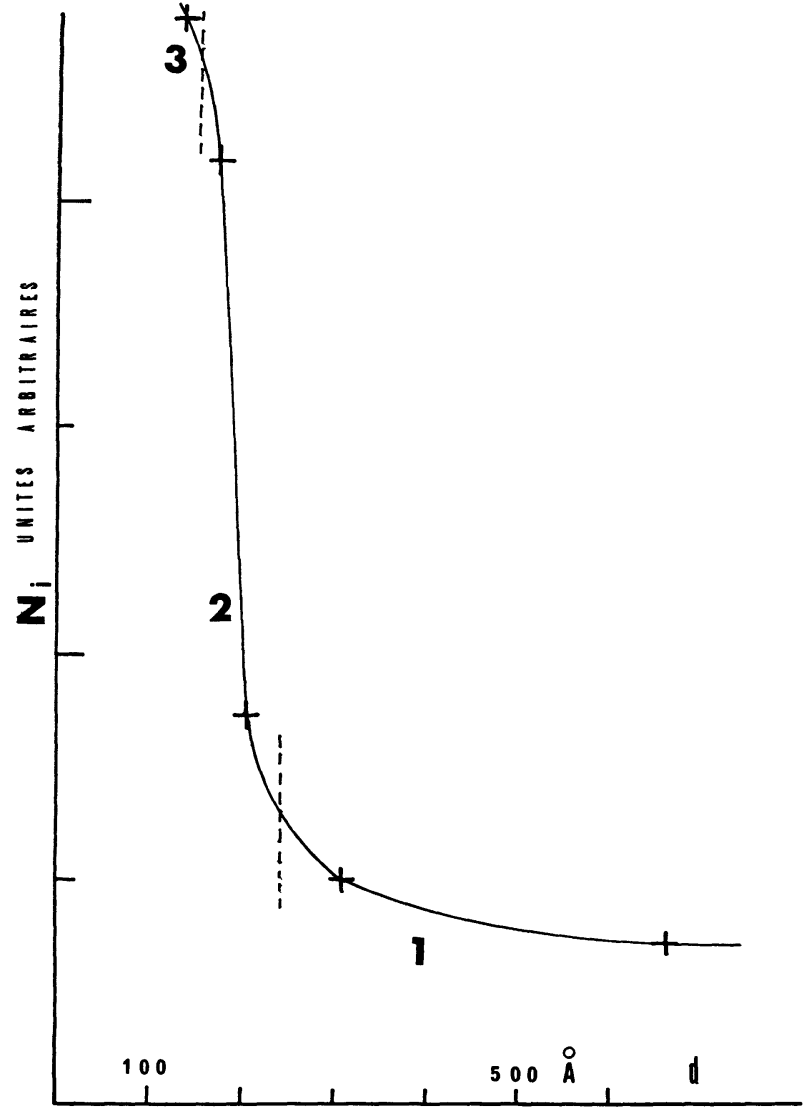

Fig. 3. - Variations de la densité de centres coulombiens $N_{\mathrm{i}}$ en fonction de l'épaisseur du diélectrique $d(\AA)$.

La croissance s'effectue ensuite sur des sites délocalisés, avec la structure de la sphalérite dans le cas du sulfure de zinc. La densité d'impuretés $N_{\mathrm{i}}$ observée au-dessus de l'épaisseur critique peut correspondre à l'insertion de particules ionisées provenant du gaz réactif ; cet effet est alors inhérent au processus de pulvérisation.

4.2 Conduction ohmiQue a FAIBLe Champ. - A faible champ électrique $\left(E<10^{5} \mathrm{~V} / \mathrm{cm}\right)$ la conduction est ohmique et permet de définir une conductivté, variable avec l'épaisseur dont l'expression est représentée par

$$
\sigma=\sigma_{0}+\sigma_{1} \exp ^{-b d}
$$

avec $d$ : épaisseur du diélectrique

$b:$ pente de la droite $\log \frac{\sigma-\sigma_{0}}{\sigma_{1}}$ en fonction de $d$ pour les faibles épaisseurs.

$\sigma_{0}$ représentant la conductivité limite observée au-dessus de $500 \AA$ et $\sigma_{1} \exp ^{-b d}$ une conductivité tunnel, compatible avec les équations de Simmons [10].

Les variations dimensionnelles de $\sigma$ et les variations thermiques du courant de conduction [4] ont permis de conclure à un courant, résultant non d'une émission Poole-Frenkel pure, mais d'une émission tunnel thermiquement assistée, à travers les barrières coulombiennes réparties dans le diélectrique.
La figure 4, représentative des variations de $\sigma$ avec $d$, présente, comme la figure 3 une brusque variation et un changement qualitatif de la cinétique de conduction pour l'épaisseur critique de $150 \AA$, compatible avec l'hypothèse d'une augmentation de la densité des centres coulombiens.

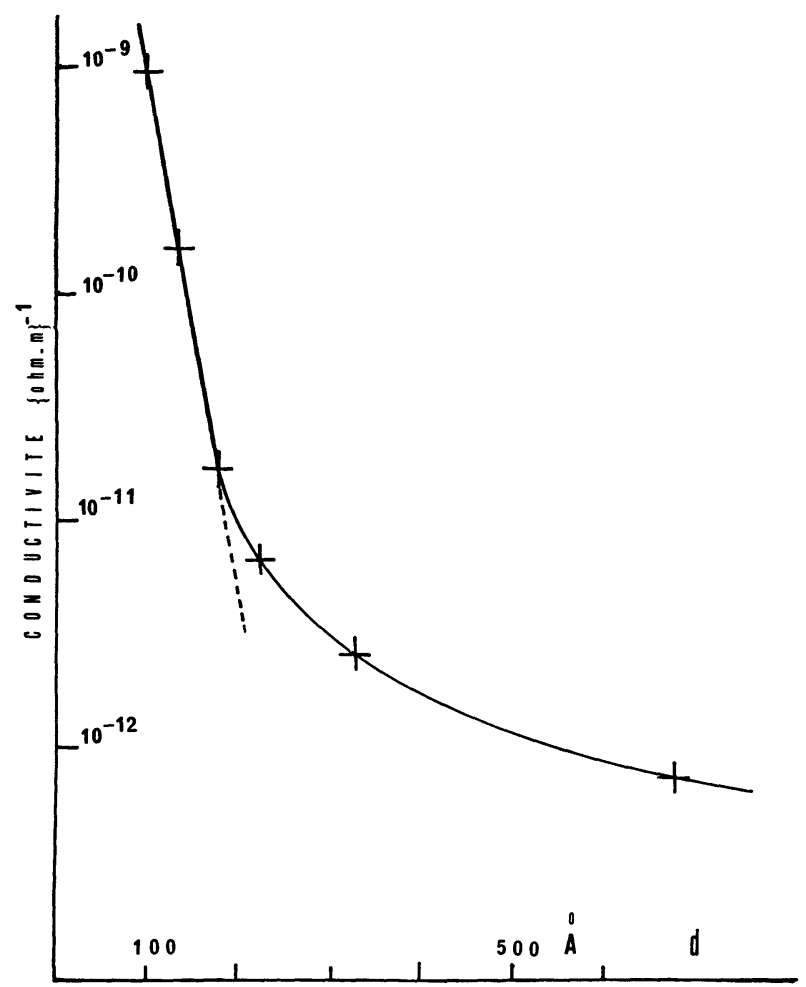

FIG. 4. - Variations de la conductivité ohmique en fonction de l'épaisseur du diélectrique $d(\AA)$.

D'autre part, de façon identique à l'étude des paramètres diélectriques, nous constatons l'homogénéité du sulfure de zinc, au-delà de $500 \AA$ avec une valeur de la conductivité voisine de celle du sulfure de zinc massif [4].

5. Effets photovoltaïques. - Les effets photovoltaïques, étudiés précédemment [2] se caractérisent par une tension en circuit ouvert et un courant de court-circuit (Fig. 5) présentant un point d'inversion en fonction de la longueur d'onde du rayonnement lumineux. Cette inversion est attribuée à deux effets antagonistes :

- des transitions interbandes lorsque l'énergie des photons est supérieure à la largeur de la bande interdite $(3,66 \mathrm{eV})$;

- une photoexcitation des centres coulombiens qui subsiste seule lorsque l'énergie des photons est inférieure à $E_{\mathrm{g}}(3,66 \mathrm{eV})$.

A partir des résultats expérimentaux, nous avons pu donner la formulation mathématique de ces deux effets [2]

$$
I_{\mathrm{TI}}=G_{\mathrm{ph}} V+I_{\mathrm{pho} 2}
$$




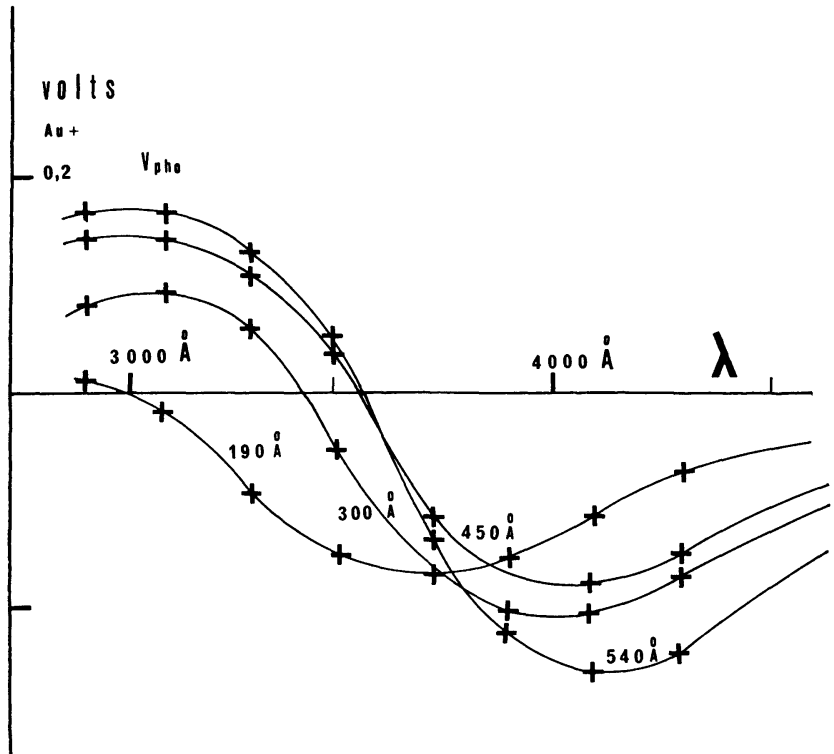

Fig. 5. - Variations de l'effet photovoltaïque avec la longueur d'onde $\lambda$.

pour les transitions interbandes et

$I_{\mathrm{PF}}=I_{0}\{1+K(F, v)\} \exp \frac{\beta}{k T}\left(V-\frac{V_{\mathrm{pho}}}{2}\right)^{1 / 2}+\frac{I_{\mathrm{pho} 1}}{2}$

pour la photoexcitation Poole-Frenkel

Le point d'inversion du courant de court-circuit total, ou de la tension photovoltaïque correspond à l'égalité de courants de ces deux types dans un domaine d'épaisseur situé entre 240 et $460 \AA$ Å.

$I_{\mathrm{pho} 2}=I_{0}\{1+K(F, v)\} \exp \frac{\beta}{k T}\left(-\frac{V_{\mathrm{pho}}}{2}\right)^{1 / 2}+\frac{I_{\mathrm{pho} 1}}{2}$.

L'égalité :

$$
I_{\text {pho } 1}=2 I_{0}(1+K) \exp \frac{\beta}{k T}\left(-\frac{V_{\text {pho }}}{2}\right)^{1 / 2}
$$

vérifiée expérimentalement, ainsi que les dépendances linéaires de $I_{\text {pho } 1}$ et $I_{\text {pho } 2}$ avec le flux lumineux, entrâ̂nent l'indépendance du point d'inversion en fonction $\mathrm{du}$ flux lumineux. Ce point d'inversion $\lambda_{0}$ est donc intrinsèque à l'épaisseur du diélectrique.

$\mathrm{Si}$ on représente (Fig. 6), les variations de $h v_{0}=h c / \lambda_{0}$ en fonction de l'épaisseur du diélectrique, nous obtenons une courbe qui rend compte des variations dimensionnelles des deux effets photovoltaïques. Celle-ci présente un aspect qualitatif cohérent avec les résultats obtenus en conduction c'est-à-dire :

- une photoexcitation Poole-Frenkel renforcée pour les faibles épaisseurs qui détermine seule un renforcement spécifique de la distorsion de la bande de conduction pour ces faibles épaisseurs (Fig. 5) ;

- une caractéristique intrinsèque du sulfure de zinc, à savoir, la valeur limite de $h v_{0}$, proche de la largeur de la bande interdite du sulfure de zinc cubique.

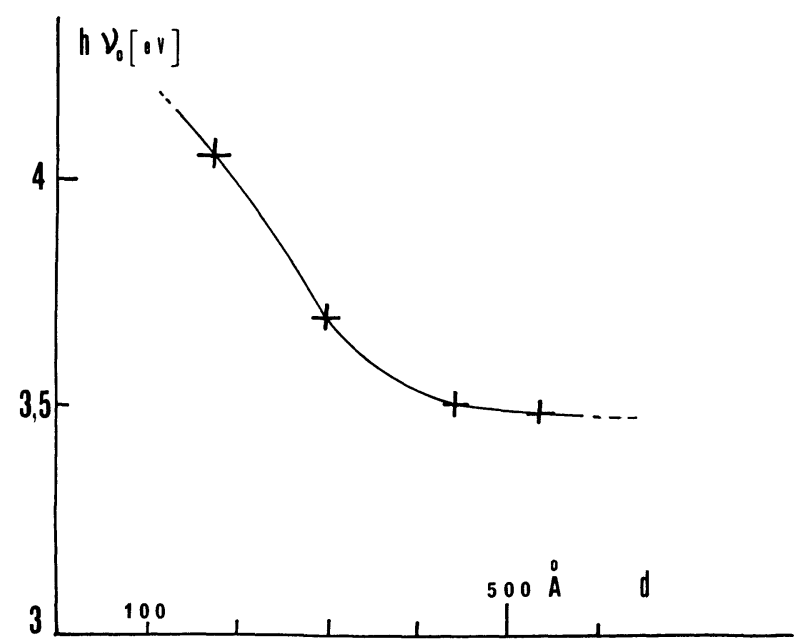

FIg. 6. - Variations du point d'inversion $h v_{0}$ de l'effet photovoltaïque avec l'épaisseur du diélectrique $d(\AA)$.

6. Discussion. - Si un grand nombre de travaux concernent les couches minces pulvérisées [11], les phénomènes de croissance ont fait l'objet d'une étude exhaustive particulièrement complète pour les couches métalliques et diélectriques obtenues par pulvérisation cathodique en champ continu [3].

L'étude que nous présentons permet d'établir un modèle macroscopique de croissance de couches pulvérisées par un champ électrique haute fréquence. Cette étude suppose, dans le cas des diélectriques, une définition correcte des paramètres physiques liés à la structure du dépôt. Ce n'est qu'après avoir mis au point les processus de stabilisation de l'électrode de base et du diélectrique tels que les structures obtenues présentent des caractéristiques reproductibles qu'on peut envisager une étude structurelle jusqu'aux épaisseurs diélectriques voisines d'une centaine d'Angströms.

Contrairement aux couches diélectriques obtenues par pulvérisation continue [3], les paramètres diélectriques ne permettent pas de faire apparaître les phénomènes dimensionnels périodiques, mais au contraire, montrent le caractère particulièrement homogène de celui-ci [1].

Les phénomènes dimensionnels n'apparaîssent qu'au second degré, lorsqu'ils induisent une émission électronique directement dépendante de la répartition des centres coulombiens dans le volume du diélectrique.

Le dépôt de couches minces pulvérisées en milieu réactif résulte, en tension continue ou haute fréquence de la succession des mécanismes élémentaires suivants :

- Impact d'ions positifs sur la cathode et éjection d'atomes métalliques.

- Excitation et réaction de ces atomes au sein du plasma.

- Condensation du composé sur le substrat. 
En reprenant les théories de germination croissance introduites par Laville Saint-Martin [3] nous sommes amenés à prévoir les mêmes hypothèses lors du dépôt des entités réactionnelles, mais les différences dans les conditions expérimentales (champ électrique HF, pression environ vingt fois plus faible) modifient complètement la nature des réactions qui se produisent dans le plasma ainsi que l'équilibre thermodynamique des zones entitées, puits de potentiel lors de la germination.

L'absence de phénomènes électroniques particuliers à l'interface $\mathrm{AL} / \mathrm{ZnS}$ montre que le support métallique est très rapidement recouvert par un diélectrique dont la taille des cristallites est d'environ $50 \AA$ [2]. Les différences dans les paramètres de mailles entre l'aluminium (hexagonal) et le sulfure de zinc (cubique) induisent une zone de transition dont les défauts cristallins permettent l'introduction de particules intersticielles (atomes ou ions). Celles-ci introduisant une relaxation dipolaire et renforcent la conduction Poole-Frenkel.

L'homogénéité du diélectrique observée au-dessus de $200 \AA$ résulte donc d'un dépôt qui s'effectue à partir d'un équilibre permanent entre le nombre de particules condensées et la progression des sites de germination.

7. Conclusion. - L'utilisation de la pulvérisation cathodique pour le dépôt de l'électrode de base et du diélectrique présente bien les avantages spécifiques que laissent prévoir les conditions théoriques relatives à cette méthode particulière de dépôt sous vide. Les couches obtenues présentent après stabilisation, sous l'action de variations cycliques de température et d'un champ électrique appliqué, une parfaite repro- ductibilité des caractéristiques diélectriques et électroniques en ce qui concerne les paramètres physiques extérieures; en particulier, cette stabilité des structures n'est pas affectée par des séjours à l'air ambiant ; au stade de nos expériences, des couches qui ont séjourné à l'air libre depuis deux années conservent toujours les mêmes caractéristiques de conduction et de photoconduction sans qu'une dispersion, supérieure à la précision des mesures ait pu être observée.

Les propriétés diélectriques, ainsi que les processus de conduction conduisent à l'élaboration d'un modèle dimensionnel de croissance en accord avec les premières hypothèses de Laville Saint-Martin [3].

Le dépôt du diélectrique sur l'électrode de base par pulvérisation conduit à une épaisseur critique de première espèce d'environ $150 \AA$, de type lacunaire, favorisant la présence d'impuretés provenant soit des gaz résiduels de l'enceinte, soit du gaz réactif ; au-delà de cette zone, la croissance est homogène et la couche présente les propriétés macroscopiques du matériau massif [1], [4].

Nous avons dans cette étude, regroupé l'ensemble des phénomènes conduisant à la mise en évidence de la zone de transition entre le métal et le diélectrique homogène.

On peut noter d'une part la parfaite stabilité des structures lorsque l'épaisseur dépasse la zone de transition; ceci laissant prévoir des applications possibles en microélectronique et d'autre part les propriétés photovoltaïques de cette première zone, où l'effet Poole-Frenkel se trouve amplifié. Ceci nous fait envisager la réalisation de dispositifs multicouches reproduisant, à partir d'une couche métallique très mince un modèle périodique avec une forte densité de centres coulombiens.

Bibliographie

[1] Murray, H., Tosser, A., Thin Solid Films 17 (1973) 75-83.

[2] Murray, H., Tosser, A., Thin Solid Films 24 (1974) 165-180.

[3] Laville Saint-Martin, Thèse de doctorat d'Etat ès sciences physiques Strasbourg (1969).

[4] Murray, H., Tosser, A., Thin Solid Films 22 (1974) 37-44.

[5] Frolich, H., Theory of dielectrics (Oxford University Press, Fair Lawn N. J.) 1948.

[6] Cole, K. S. et Cole, R. H., J. Chem. Phys. 9 (1941) 341.
[7] Geversm, Philips Res. Rep. 1 (1946) 279.

[8] HiLL, R. M., Thin Solid Films 1 (1967) 39-69.

[9] HiLl, R. M., Phil. Mag. 23 (1971) 59-86.

[10] Simmons, J. G., J. Appl. Phys. 34 (1963) 2581-90.

[11] Premier Colloque International de pulvérisation cathodique C. I. P. 73 Société Française du vide, Montpellier 25 octobre 1973 France.

Laville Saint-Martin, B., Collange, A., p. 241, РомPEI, J., 321. 